\title{
TURBULENCE MODEL EXTENSION FOR VORTEX DOMINATED FLOWS AND OPTIMIZATION WITH EXPERIMENTAL DATA
}

\author{
M. MOIOLI ${ }^{1}$, C. BREITSAMTER ${ }^{2}$ AND K. A. SØRENSEN ${ }^{3}$ \\ ${ }^{1}$ Chair of Aerodynamics and Fluid Mechanics, Technical University of Munich, Garching, 85748, \\ Germany, matteo.moioli@aer.mw.tum.de \\ ${ }^{2}$ Chair of Aerodynamics and Fluid Mechanics, Technical University of Munich, Garching, 85748, \\ Germany, christian.breitsamter@aer.mw.tum.de \\ ${ }^{3}$ Airbus Defence and Space, Manching, 85077, Germany, kaare.sorensen@airbus.com
}

Key words: Turbulence, Modeling, Optimization, Data-Driven Models, Vortex Dominated Flows, Delta Wing, RANS, Artificial Neural Network

\begin{abstract}
This document provides information and instructions for preparing a Full Paper to be included in the Proceedings of 14th WCCM - ECCOMAS CONGRESS 2020.
\end{abstract}

\section{INTRODUCTION}

Wing planforms which are employed for high agility aircraft consist for the major part of highly swept wings. This is the case of delta wings whose aerodynamic characteristics are dominated by the separation of large-scale leading-edge vortex flows. The vortex type and stage depends on several factors, both geometrical and related to the flow conditions. The numerical simulation of such a complex type of flow remains a source of issues for the turbulence models available at the state of the art. This is true, in particular, for the turbulence models which close a Reynolds-averaged Navier-Stokes (RANS) formulation with a Boussinesq assumption.

On the one hand, the employment of more complex and/or scale resolving turbulence models remains prohibitive with respect to the additional computational cost which is a relevant factor along the different steps of the design and development phases of a aircraft where the investigation of several variations of a complex aircraft is required. Moreover, the improvement in terms of accuracy is not always guaranteed by the additional modeling complexity and the introduction of numerical stability issues or setup complexity is also to be considered $[1,2]$.

On the other hand, the most common eddy viscosity models (EVM) [3] provide an inherent lack of accuracy with regard to highly separated and/or rotational flows where the Boussinesq assumption ceases to be a valid simplification for most cases. At the state of the art, a series of corrections for the baseline EVMs exist which address the limitations of the models for complex flows. However, these corrections are calibrated using generic test cases in order to achieve a global formulation (Fig. 1). This means the modeler prioritizes the globality and the model's validity over the widest range of cases while it sacrifices its maximal possible accuracy level which could be achieved with the calibration performed over a single point, i.e. one test case at one flow condition. The proposed methodology has the objective to 
combine the introduction of additional terms in the turbulence model equation with an automatic calibration procedure with experimental data as reference [4]. The objective is to find the best compromise between the accuracy enhancement provided by the additional model terms and a regional calibration which sacrifices only part of the globality of the baseline model.

A series of modeling corrections or extensions are available and have been formulated, a part of these are formulated with the objective of maintaining the highest grade of globality with a certain flow feature as target, for instance the Rotation-Correction of the Spalart-Allmaras model [5], whereas others consist of the maximization of accuracy for a certain target feature [6]. Therefore, the proposed methodology has the objective to exploit the best compromise between the two possible modeling directions.
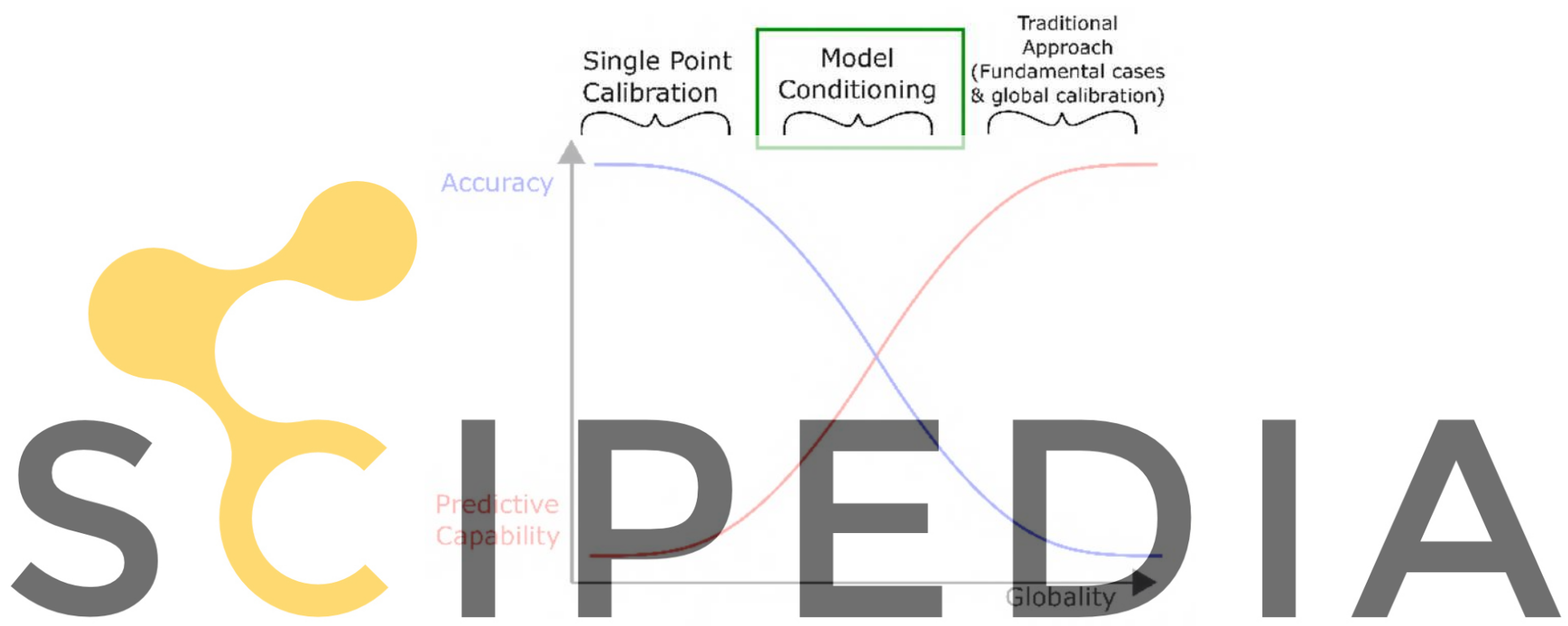

Figure 1: Qualitative visualization of the dependence of the accuracy and predictive capability levels of

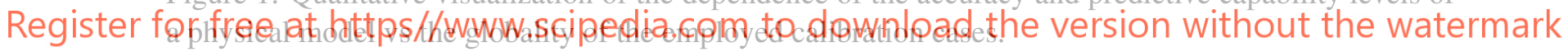

A relevant drawback of the methodology is that it relies on experimental data for the calibration of a model valid for a class of flows of a certain extension. When new cases are addressed, if the geometries or flow conditions provide low to mild differences from past calibration cases, the modeler may apply the same optimized model based on the experience and investigation of the present class of flow. However, if the new case has large differences to the previous calibration cases and there are no available experimental data, the user may avoid the employment of the methodology.

A recent research path in physical modeling in fluid dynamics consists of the integration of machine and deep learning methods to exploit of the availability the large amount of available high fidelity data to enhance the model performance [7]. In this work, a machine learning extension of the proposed methodology is also formulated with the objective to extend the predictive capability to flow cases where no experimental data are available. In the context of this work, an additional methodology is introduced in order to solve the problem. The idea is to utilize all the available data from the previous optimization procedures to correlate the geometrical and flow condition features with the resulting turbulence model 
parameters. A set of the available data is established and an artificial neural network is trained in order to predict the best set of coefficients based on the new test cases features as input.

\section{LEADING-EDGE VORTEX FLOW}

The dominating flow feature of a highly swept or delta wing is the vortex flow which separates at the leading edge. A large-scale vortex system above a delta wing may occur in different stages depending on geometrical features and flow condition factors. The more important parameters that determine the vortex flow stages are the angle of attack, the leading-edge sweep angle and the leading edge curvature. The leading edge shape plays a fundamental role on the separation onset of the vortex flow along the leading-edge extension [8]. When the leading edge is sharp, the primary vortex flow separation is fixed whereas at a blunt leading edge the primary separation strongly depends on the Reynolds number. With regards to compressibility effects, Mach number plays the major role. The variation of the vortex flow stage with an increase of the angle of attack is for a certain grade comparable to a decrease of the sweep angle (Fig. 2) [9].

Starting from small angles of attack, no vortex may be present above the wing surface and this is particularly true if the leading edge is characterized by a small curvature. The flow is able to remain attached to the wing surface. As the angle of attack increases, the separation of the flow generates a vortex sheet which rolla up above the wing into a vortex of stable structure. The vortex structure is characterized by high tangential and axial velocities which interact with the upper wing surface and this causes an induced suction footprint that determines the additional vortex lift $[9,10]$. The axial velocities may reach values

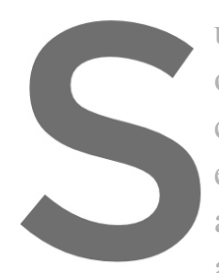
up to three times the freestream velocity in the vortex core. The flow directed under the nain vortex and
outboard to the leading edge may separate in a secondary vortex. This is a viscous phenonenon and is
caused by the strong adverse pressure gradient between the yortex suction peak and the wing's leading
edge [11]. In the case of a rounded leading edge, at low angles of attack, the separation takes place
along a certain portion of the leading edge and a partially developed vortex is present. As the angle of
attack increases, the separation onset moves upstream until it reaches and remains at the apex. By further increasing the angle of attack, the vortex changes its axis direction which moves inwards. Register for free at https//www. scipedia.com to download the version without the watermark abrupt change of the vortex flow field takes place. This instability phenomenon is called vortex break down and it causes the vortex to lose its structured and helicity dominated nature into a wake like and chaotic flow [12]. Hence, a drop in the vortex induced tangential and axial velocity is encountered which determines a sudden change in the aerodynamic forces. The angle of initial breakdown is defined as the angle of attack at which the breakdown instability takes place above the trailing edge. As the angle of attack further increases, the breakdown moves upward until a fully burst and shedding condition is reached where basically the breakdown takes place at the apex of the wing.

\section{FORMULATION OF THE TURBULENCE MODEL ENHANCEMENT AND OPTIMIZA- TION PROCEDURE}

In the course of the Advanced Aircraft Understanding via the Virtual Aircraft Model (VitAM) Project [13], the Technical University of Munich (TUM) and Airbus Defence \& Space (ADS) have collaborated in order to improve the available numerical tools and procedures for the virtual development and design of an aircraft. Whereas efficiency has been mainly addressed with success by ADS [13], the main focus 


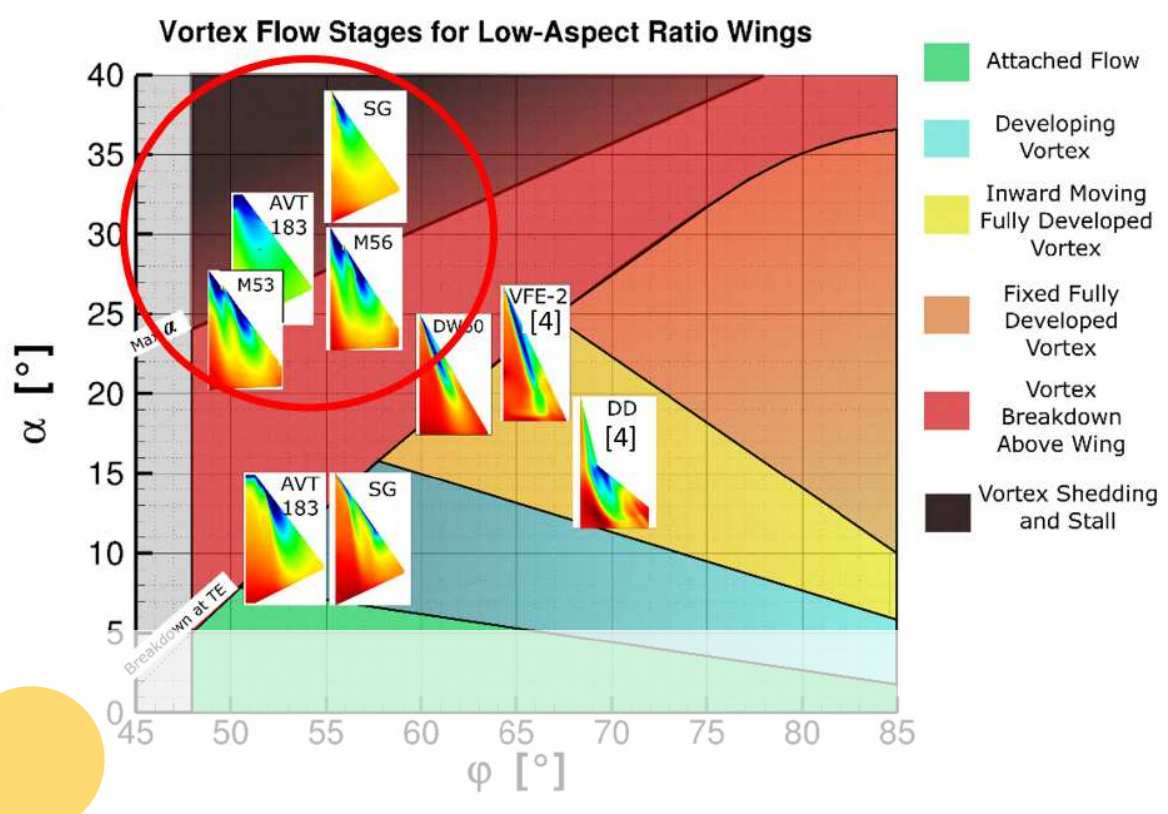

Figure 2: Qualitative representation of the vortex development stages for different leading-edge shapes and their dependence on the angle of attack $\alpha$ and wing sweep angle $\varphi$. Also shown, the positioning of the different test cases which have been investigated in the course of the VitAM project [4].
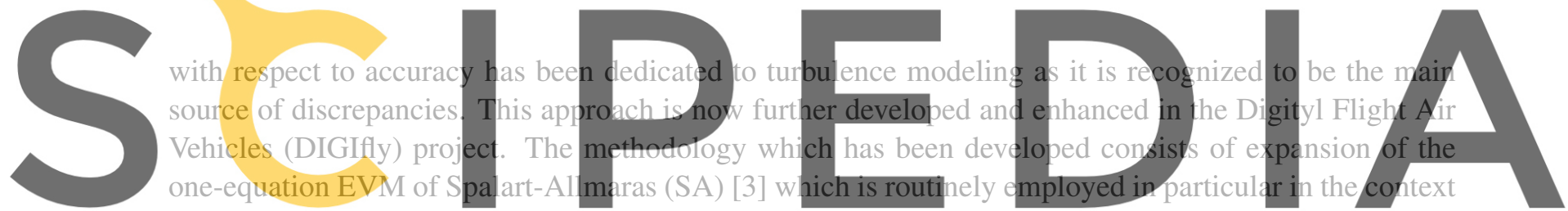

of external aerodynamic cases and it has shown throughout the years a well behavior with respect to

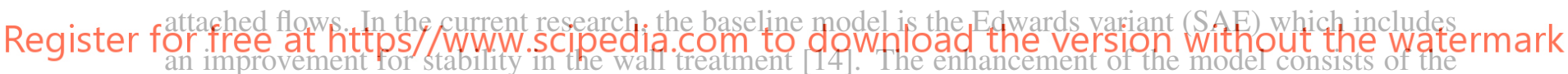
following key points [4]:

- A series of production terms are formulated in order to have different sensitivities for different types or regions of vortex flows.

- The additional terms are active exclusively inside the vortex flow field, without changing the modeling inside the boundary layer region which is already well predicted by the baseline SA model.

- The model's enhancement does not introduce significant additional computational costs or numerical instabilities.

- The model maintains the Galilean invariance property and the additional terms are non-dimensional.

In order to activate the effect of the turbulence model enhancement in the vortical region only, a vortex identifier quantity $\xi$ is formulated (Eq. 1) and coupled with every additional term. The variable $\xi$ consists of a limited ratio between the vorticity and the strain rate. The limit value $c_{v l}$ is set equal to 1.0 in order to avoid any influence on the boundary layer region. 


$$
\xi=\max \left[\left(\left(\frac{\omega}{S+\varepsilon}\right)-c_{v l}\right), 0.0\right]
$$

The turbulence model formulation is reported in the following equation (Eq. 2):

$$
\frac{D \tilde{\mathrm{v}}}{D t}=c_{b 1} \tilde{S}\left(1-f_{t 2}\right) \tilde{\mathrm{v}}-[\overbrace{c_{b v 1} \xi S \tilde{\mathrm{v}}}^{(i)}+\overbrace{c_{b v 2} \xi^{\frac{1}{2}} S \tilde{\mathrm{v}}}^{(i i)}+\overbrace{c_{b v 3} \xi^{2} S \tilde{\mathrm{v}}}^{(i i i)}+
$$

(iv)

$$
+\overbrace{c_{b v 4}\left(\min \left(\frac{1}{\xi}, c_{v r, l i m}\right)\right) \xi_{s w} S \tilde{\mathrm{v}}}+\overbrace{c_{b v h 1} \xi \tilde{\mathcal{H}} \tilde{\mathrm{v}}}^{(v)}+
$$

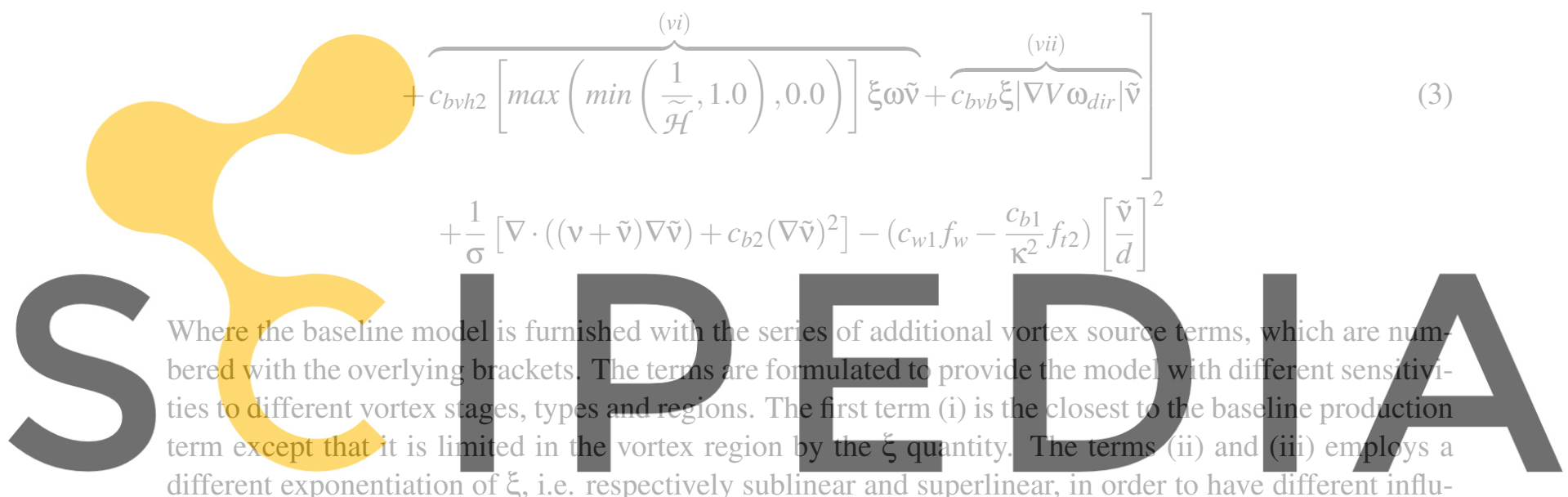

different exponentiation of $\xi$, i.e. respectively sublinear and superlinear, in order to have different influ-

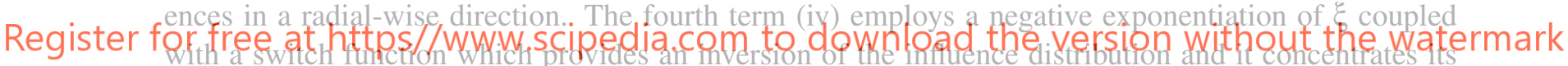

effect on the outer regions of the vortex. Whereas the first four terms are based on variations of their dis-

tribution along the radial direction, the remaining three distinguish their regions of influence according to regions relative to the breakdown position. Hence, one term (v) uses an absolute and non-dimensional helicity variable to target the highly structured and stable vortex part upstream of the breakdown instability. Consequently, an inverse term (vi) is formulated and it is coupled with a switch function based on the inverse of helicity that focuses its influence on the wake region downstream of breakdown. The last one (vii) is based on the product between the vorticity direction and the gradient of velocity, the quantity of which has high values in particular in proximity of breakdown.

The automatic calibration of the coefficients of the additional vortex source terms is performed by means of a toolchain which handles the I/O between optimization algorithm and numerical simulations as well as the monitoring of the simulations. The objective function $\varepsilon(\mathbf{c})$ of a certain set of coefficients $\mathbf{c}$ is:

$$
\varepsilon(\mathbf{c})=\frac{\sum_{i=1}^{m} \sum_{j=1}^{n}\left[\varepsilon^{(i, j)} w_{d p}^{(i)}\right]}{m n\left(\sum_{i=1}^{m} w_{d p}^{(i)}\right)}
$$


Where $n$ is the number of experimental data points, $m$ is the number of design points $(d p)$ and $w_{d p}$ is the weighting factor which can be varied for the design points. For the presented results, no particular weight function was necessary, hence the value is always set equal to 1 .

The algorithm consists of a gradient descent algorithm with a relaxation factor of 0.5 . The toolchain starts with a first simulation from an initial converged solution which can be the baseline SAE solution, i.e. $\mathbf{c}_{0}=0.0$, or a different starting set of coefficients. This may be relevant in order to investigate the inherent locality of the algorithm and improve the calibration result. After having conducted the starting point simulation a series of incremental simulations are restarted. Every incremental simulation corresponds to an increment of one of the active turbulence model parameters. This process continues iteratively until the optimization reaches a minimum of the minimization function $\varepsilon(\mathbf{c})$ and the final set of coefficients is selected as the optimized parameters of the model.

\section{OPTIMAL TURBULENCE MODEL PREDICTION BY MEANS OF ARTIFICIAL NEURAL NETWORK}

\subsection{Motivation and Idea}

Several test cases have been addressed for the application of the optimization methodology and the investigation of its performance on different types of delta wings. Due to the locality of the chosen optimization algorithm, usually different optimization procedures are tested for verifying the locality of the optimum reached. Therefore, along the project a relatively large amount of RANS simulations have been performed for the calibration procedures with different values of the turbulence model parameters.

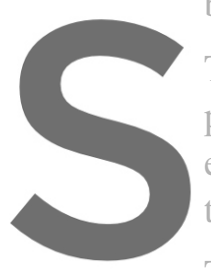
The idea underlying this extensi
procedure which is that it require
ences with the available previous
the methodology acquires low po

The test cases which have been reported in this article are highlighted with a red circle in Figure 2 . In this vortex flow map, also other. examples of test cases are included which have been investigated and

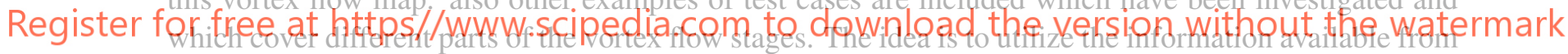

the data of all these previous optimizations and sensitivity tests in order to predict an appropriate set of coefficients for a new test case. It is reasonabie to expect that the achieved accuracy might not be at the same grade as a successful gradient descent optimization. The objective is to address how accurate is this type of prediction and how much it can increase the predictive capability of the available information.

\subsubsection{Dataset Description and Augmentation}

The dataset is therefore composed of a series of features $\mathbf{x}$ and target values $\mathbf{y}$. The features consist of the geometrical and flow condition parameters which describe the test case and its flow stage (Tab. 1). With respect to the flow conditions, the features include the Mach and Reynolds number and the AoA range. The geometric features include the sweep angle of the first and second section, $\varphi_{1} \varphi_{2}$, if a strake wing is present, the taper ratio $\lambda$, the aspect ratio $\Lambda$ and the normalized leading-edge radius $r_{L E} / c_{r}$ with $c_{r}$ as the wing root chord. The target data are the values of the turbulence model coefficients.

In the best scenario, the dataset would be composed exclusively of points which describes optimal set of coefficients, however, not enough data points are available with respect to the final optimized results. The 
Table 1: Features x.

\begin{tabular}{llllllll}
\hline Mach Reynolds AoA min & AoA max & $\varphi_{1}$ & $\varphi_{2}$ & $\lambda$ & $\Lambda$ & $r_{L E} / c_{r}$
\end{tabular}

application of a machine learning approach to a restricted database would lead to an over-fitted model which is not recommended for extensive applications. Hence, the dataset is augmented by including all the available intermediate points inside the optimization processes. In this case, the feature which describes the minimization function $\varepsilon(\mathbf{c})$ needs to be included in the features vector $\mathbf{x}$.

\subsection{Artificial Neural Network Setup, Hyper-parameters and Training}

The dataset preparation and the artificial neural network (ANN) is performed by means of the Tensorflow 2 library in a Python framework [15]. The ANN is a feed-forward multi-layer network of a series of hidden layers composed of this series of neuron numbers:

$$
[512,256,128,64,64,64,32,16,8]
$$

A LeakyReLU activation function is employed for all layers and a batch normalization method is used for regularization. In addition to that, a skip connection of the input features is concatenated to the last hidden layer. This has shown significant improvements in the training performance. All the hyperparameters are found by means of a grid search.

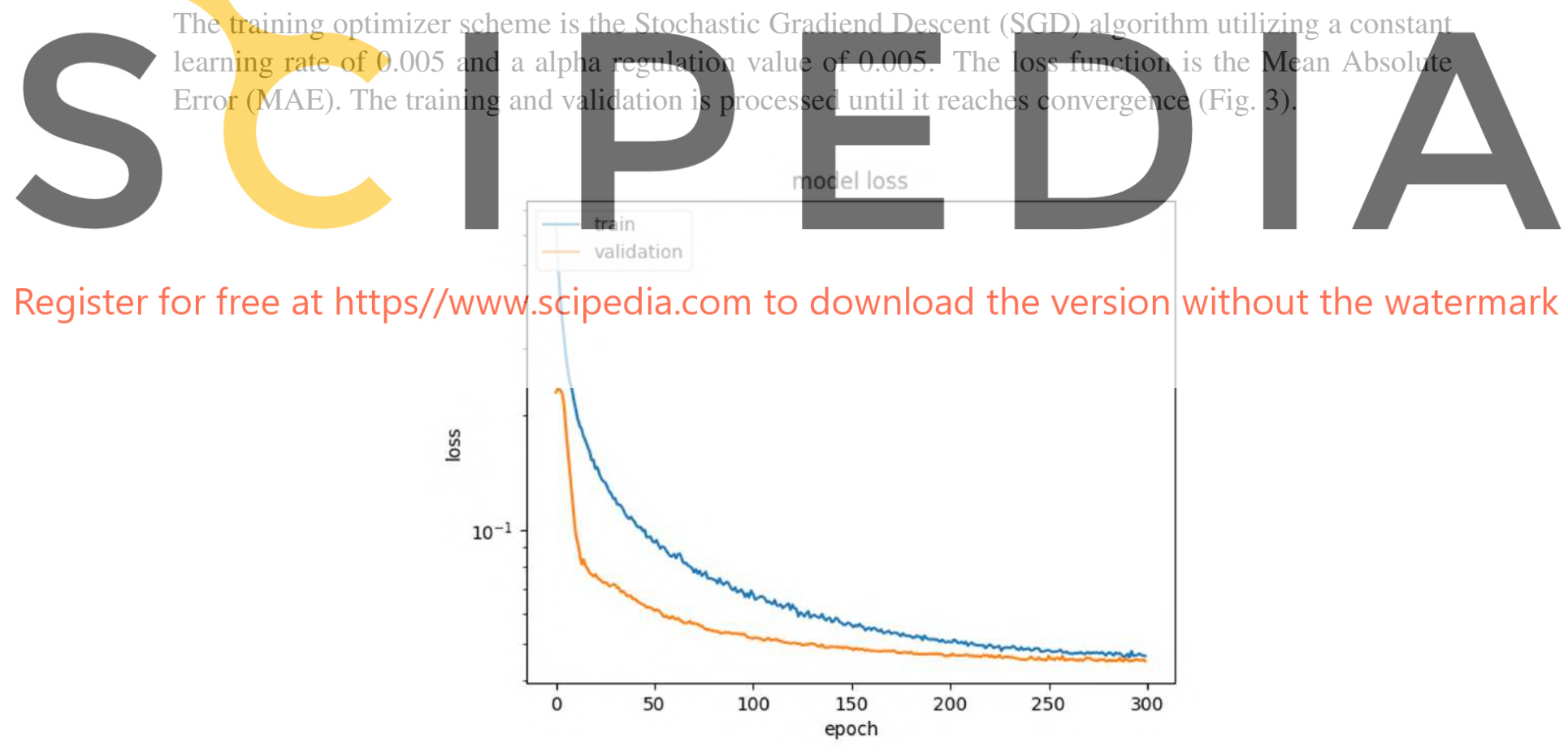

Figure 3: Loss function of the training and validation data vs the training epochs. 


\section{RESULTS AND DISCUSSION}

\subsection{Numerical Setup}

The numerical simulations in this study have been performed by means of the TAU-Code solver of the German Aerospace Center (DLR). The solver is based on a cell-vertex finite volume methodology with the capability of solving hybrid-unstructured grids. The RANS equations are solved in a fully turbulent regime, with an implicit dual-time stepping approach for the stabilization of the solver, a Multigrid relaxation scheme and an implicit Backward-Euler/LUSGS smoother. With respect to the flux discretization method, an improved version of the Advection Upstream Splitting Method (AUSMDV) is used because it combines the advantaged of the flux Difference (AUSMD) and the Vector splitting (AUSMV) variants. The geometry of the test cases reported in these studies are split into a half model because no asymmetric flow conditions are investigated. If the wind tunnel model of reference are provided with a support or peniche, these are included in the flow domain and a symmetry plane boundary condition is placed at a certain displacement from the wind tunnel floor by taking into account the corresponding boundary layer thickness. The SAE model in its baseline or optimized form is employed for all the simulations.

\subsection{Model53 and Model56}

The Model53 and Model56 (Tab. 2) consist of two sweep angle variations, i.e. of $53^{\circ}$ and $56^{\circ}$ respectively, of the same baseline geometry. The wing geometry is a generic delta wing with a deployed leading-edge slat at $-20^{\circ}$ and a tip twist of $4^{\circ}$. The deployment of the slat is a plausible scenario for the angle-of-attack range of main interest, which includes angles from medium to very high values where the main discrepancies correlated with the turbul
deployed slat increases the complexity of the flow w
ogy application. In addition to the first vorte wh
separates above the deployed slat.
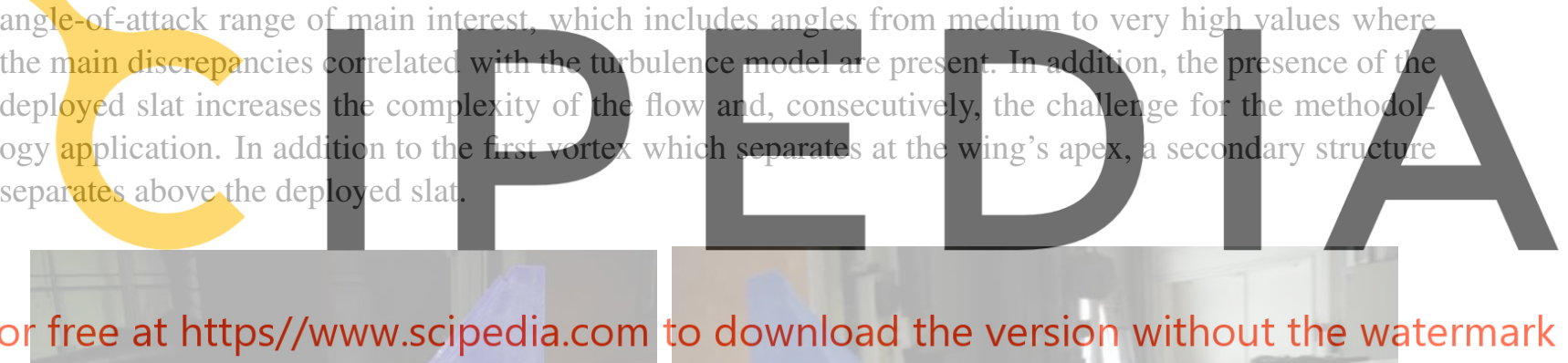

Register for free at https//www.scipedia.com

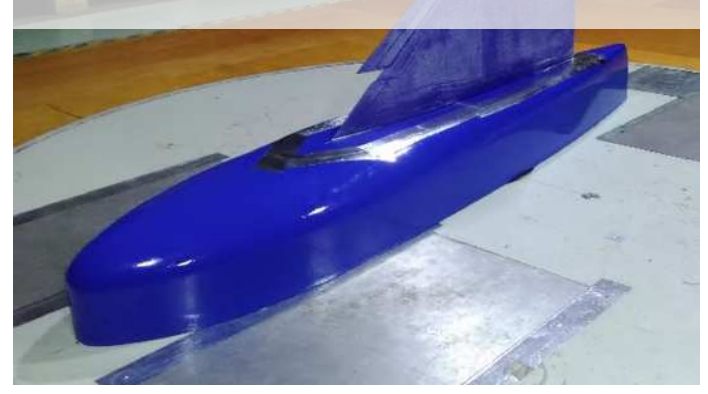

(a) Mode153

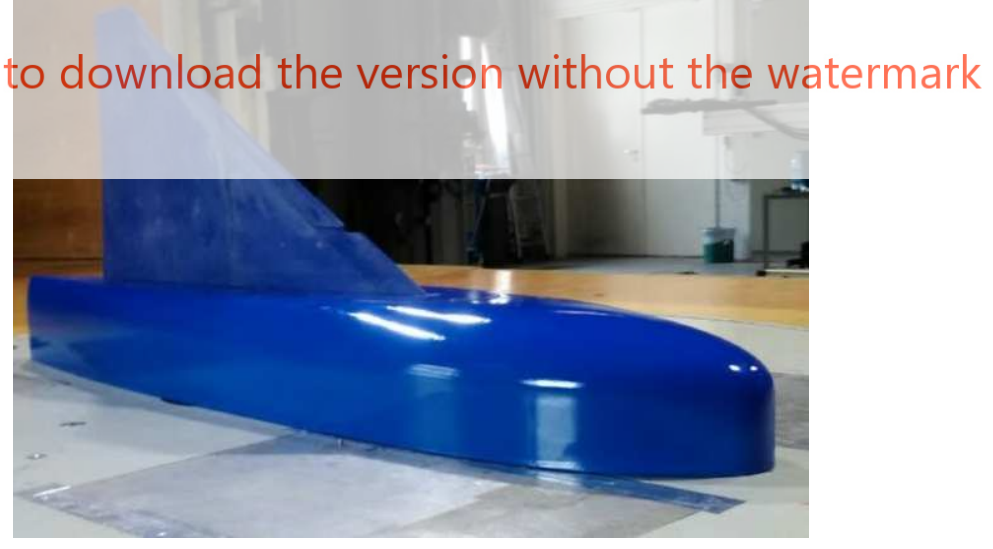

(b) Model56

Figure 4: Wind tunnel models 53 and 56 in the W/T A facility at TUM.

The wind tunnel models (Fig. 4) are produced by means of a peculiar technique which employs a additive 
manufacturing technique to produce a model provided with internal taps for the measurement of stationary surface pressures [16]. The wind tunnel models are equippied with a generic fuselage and peniche support, also produced by additive manufacturing and they are measured at the Wind Tunnel A (W/T-A) facility at TUM. The flow conditions correspond to a Mach number of 0.15 and a Reynolds number of $1.7 \cdot 10^{6}$ based on the mean aerodynamic chord. A wide range of angles of attack have been measured but for the information required in this work, only the angle of attack with the largest discrepancy between the numerical and experimental data is reported which is $A o A=28$.

Table 2: Model53/56: Wing geometry parameters.

\begin{tabular}{c|ccccccc}
\hline Model & $c_{r}[m]$ & $c_{t}[m]$ & $\lambda$ & $\varphi_{T E}$ & $S_{r e f}\left[m^{2}\right]$ & $\Lambda$ & $l_{\mu}[m]$ \\
\hline Mode153 & 0.75 & 0.12 & 0.16 & $-2.7^{\circ}$ & 0.204 & 2.06 & 0.51 \\
Model56 & 0.75 & 0.11 & 0.146 & $-2.7^{\circ}$ & 0.182 & 1.92 & 0.505 \\
\hline
\end{tabular}

The availability of a sweep angle variation of the same geometry provides the possibility to perform the calibration process on one sweep angle, and then, validate the optimized model on the other. Hence, first of all the accuracy improvement is analyzed on the calibration case and, secondly, the predictive capability is checked by applying the same turbulence model to the validation case. This is of major importance for yerifying that the methodology is able to furnish a model which extends its accuracy enhancement to a certain range of variations from the calibration case. Being the sweep angle a sensitive parameter with regards to the vortex type and stage, this validation delivers important information about the well
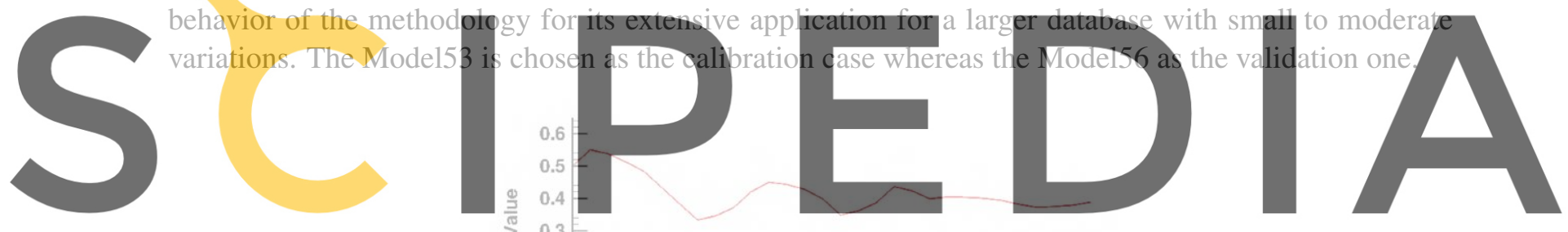

Register for free at https//ww商.scipedia.com to download the ve्chsion without the watermark

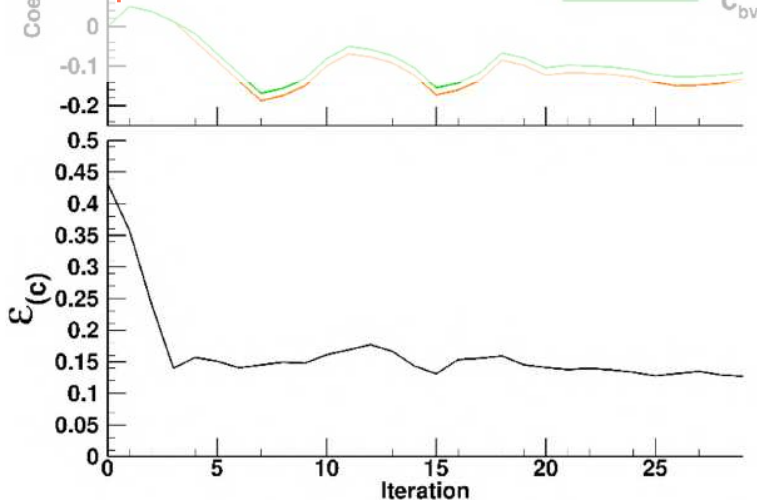

Figure 5: Optimization convergence of the objective function and the turbulence model coefficients along the iterations of the procedure for the Model53 at $\mathrm{AoA}=28^{\circ}$.

The calibration procedure is performed on the angle of attack of $28^{\circ}$ and it provides a significant im- 
Table 3: Optimized set of coefficients for the Model53.

\begin{tabular}{c|ccc}
\hline Coefficient & $c_{b v 1}$ & $c_{b v h 1}$ & $c_{b v h 2}$ \\
\hline Value & 0.41 & -0.11 & -0.10 \\
\hline
\end{tabular}

provement of the mean average error of the surface pressure coefficient distribution (Fig. 5). The final set of coefficients is selected as optimized model and it includes three parameters of the additional turbulence model terms (Tab. 3). The terms which have been excluded for the calibration have indicated a low sensitivity, similar to other terms during the preliminary sensitivity testing and other optimization procedures derived from different starting points for addressing the locality. Compared to the baseline SAE model, the improvement is significant for different angles of attack for both the calibration (Tab. 4) and the validation cases (Tab. 5). This shows the potential of the methodology to improve a certain extension of cases around the calibration case with a significant accuracy improvement.

Table 4: Mean absolute error between numerical and experimental surface pressure data for the Model53.

\begin{tabular}{c|cc|c}
\hline $\mathbf{A o A}^{\circ}$ & SAE model & Optimized & Difference \\
\hline 25 & 0.1570 & 0.1348 & $-14.1 \%$ \\
28 & 0.4253 & 0.1411 & $-66.8 \%$ \\
\hline
\end{tabular}

Table 5: Mean absolute
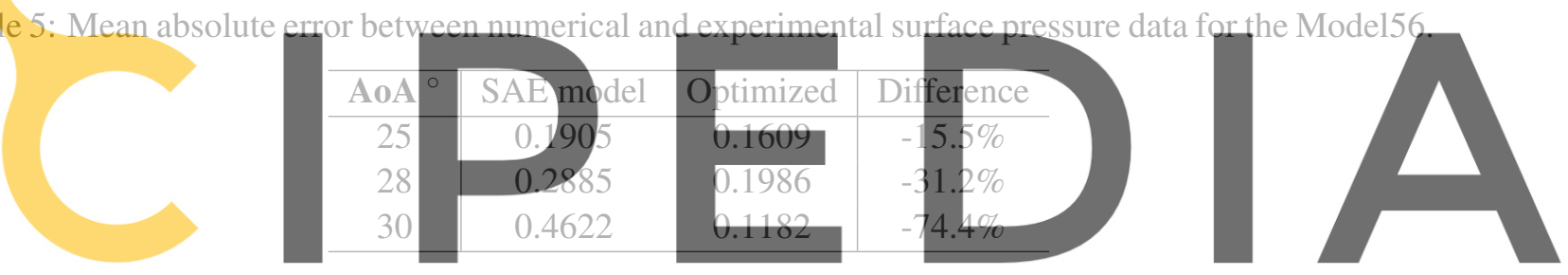

The surface pressure distribution of the wing for the calibration case at $A \circ A=28$ shows a significant

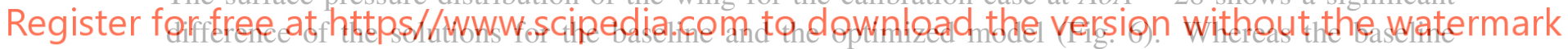

SAE model predicts a developed vortex downward nearly $60 \%$ of the wing root chord where breakdown occurs, the optimized model returns a fully stalled and vortex shed condition. Therefore, the grade of accuracy with the experimental data is improved. Instead of a suction peak, a flat distribution is visible above the complete wing providing a significant match with the experimental data. The available pressure data on the lower wing surface indicate how the sensitivity of the vortex source terms is present only in the vortex flow field. In fact, there is no significant variation between the two turbulence models and they both match with the experimental data on the lower surface where an attached flow is present. The two apex and slat vortices are of different nature because of their different effective angles of attack and the different geometrical contour that the flow has to follow. The interaction between the two vortices shows how the breakdown of the less stable apex vortex triggers the slat vortex to burst and merge into a common chaotic wake. The suction level downstream of breakdown has a certain offset to the experimental data when the optimized model is employed. In general, the accuracy improvement is significant (Tab. 4) and the optimized model is chosen as an optimal set of coefficients to be applied for similar test cases of non-slender delta wings, i.e. with a sweep angle in the proximity of $53^{\circ}$.

For the Model56 treated as validation case the same AoA as for the Model53 calibration case, namely 


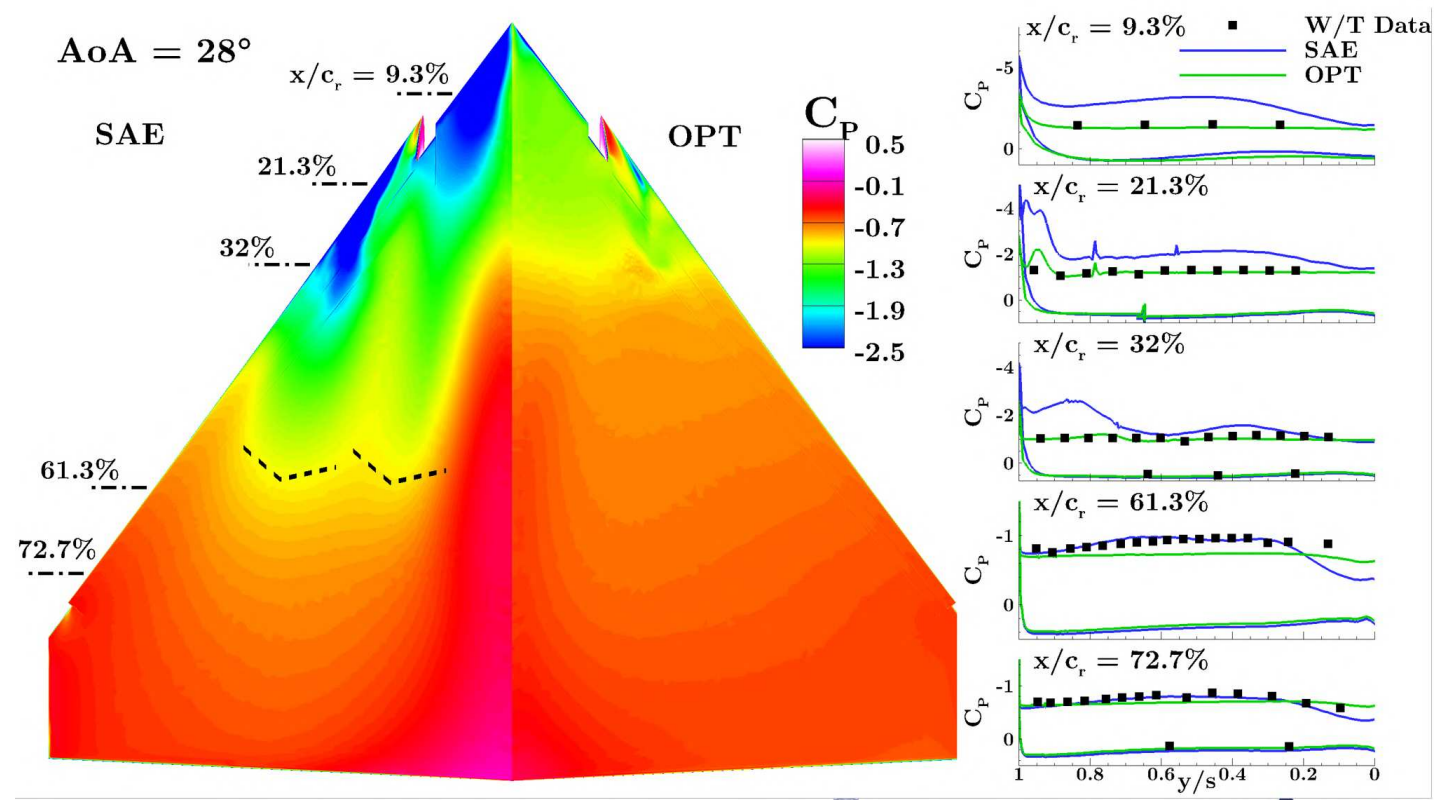

Figure 6: $C_{P}$ surface contour plots and sectional $C_{p}$ distributions of the Model53 at the flow conditions of $\mathrm{M}=0.15, \mathrm{Re}=1.7 \cdot 10^{6}$ and $A o A=28$. The numerical results of the baseline (SAE) and the optimized model (OPT) (Tab. 3) are compared to the experimental data (W/T Data).
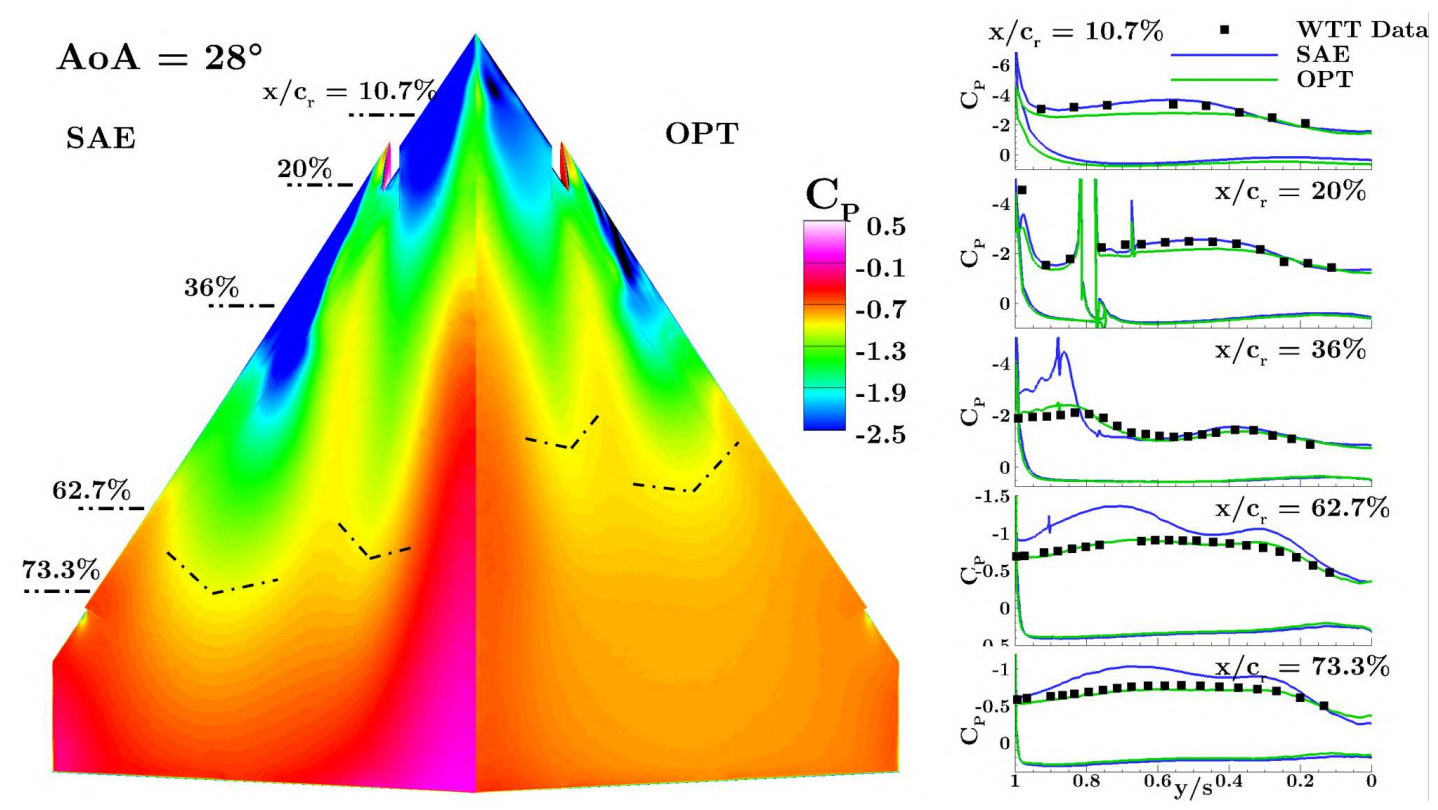

Figure 7: $C_{P}$ surface contour plots and sectional $C_{p}$ distributions of the Model56 at the flow conditions of $\mathrm{M}=0.15, \mathrm{Re}=1.7 \cdot 10^{6}$ and $A o A=28^{\circ}$. The numerical results of the baseline (SAE) and the optimized model (OPT) (Tab. 3) are compared to the experimental data (W/T Data). 
AoA $=28^{\circ}$, is chosen in order to show the significant improvement achieved with a different flow field from the calibration case. In this case, the experimental data indicate that no stall condition is reached. Instead a fully developed vortex with breakdown instability is present, however, the breakdown position is much upstream compared to the SAE numerical result. The application of the optimized model provides an accurate match with experimental data (Tab. 5)

\subsection{Extension of the Optimized Model to other Test Cases}

The optimized model on the Model53 is used in the following section for the cluster of cases highlighted in Figure 2 which share a similar sweep angle but significant differences for other features.

The extension of the optimized model (Tab. 4) is firstly performed as a direct application to the AVT-183 diamond wing configuration and a Blended Wing Body test cases. This approach is usually performed when the modeler has the availability of an optimized model for a case with relatively moderate differences in terms of the vortex flow field evolution.

Secondly, the ANN methodology described in Section 4 is applied for the more interesting angle of attack for additional test cases in order to evaluate the potential of the method to extend the predictive capability of the available calibrated turbulence models.

\subsubsection{AVT-183 Configuration}

The AVT-183 test case consists of a $53^{\circ}$ swept diamond wing and it has been the target of the investigation performed in the NATO-STO AVT-183 task group [17, 18]. The geometry has a rounded leading-edge shape which causes partially developed vortex stages in the range of smaller angles of attack. The research task group selected as target feature the prediction of the separation onset with the numerical simulations. However, data are available also for larger angles of attack which are employed in this work for the verification of the turbulence model optimized on the Model53. The flow conditions are a Mach number of 0.15 , i.e. equal to the Model53, and a Reynolds number of $2.7 \cdot 10^{6}$ based on the mean aerodynamic chord. Except for the sweep angle which is also $53^{\circ}$, different geometrical features are present compared to the Model53 (Tab.6). Moreover, the wind tunnel geometry is also a half model but it is mounted on a peniche without a generic fuselage which may influence differently the development of the vortex system. In addition, no deployed slat geometry is present, hence a singular vortex flow separates at the wing's leading edge. Therefore, the test case provides a challenging benchmark for the extension of an available optimized model.

Table 6: AVT-183: wing geometry parameters.

\begin{tabular}{ccccccccc}
\hline$c_{r}[m]$ & $c_{t}[m]$ & $\lambda$ & $\varphi_{L E}$ & $\varphi_{T E}$ & $S_{r e f}\left[m^{2}\right]$ & $\Lambda$ & $l_{\mu}[m]$ & $r / c_{r}$ \\
\hline 1.2 & 0.0 & - & $53^{\circ}$ & $-26.5^{\circ}$ & 0.394 & 2.191 & 0.8 & 0.246 \\
\hline
\end{tabular}

With respect to an AoA of $28^{\circ}$, the numerical results with the baseline SAE model provides a fully developed vortex with breakdown (Fig. 8). The breakdown location appears to be between $x / c_{r}=0.1$ and 0.2 where a significant drop in the suction values is present and downstream of that a flat distribution dominates the wing surface. The development of the suction distribution along the cross-flow sections (Fig. 9= is comparable to the Model53 case but the breakdown location is much more upstream. The 


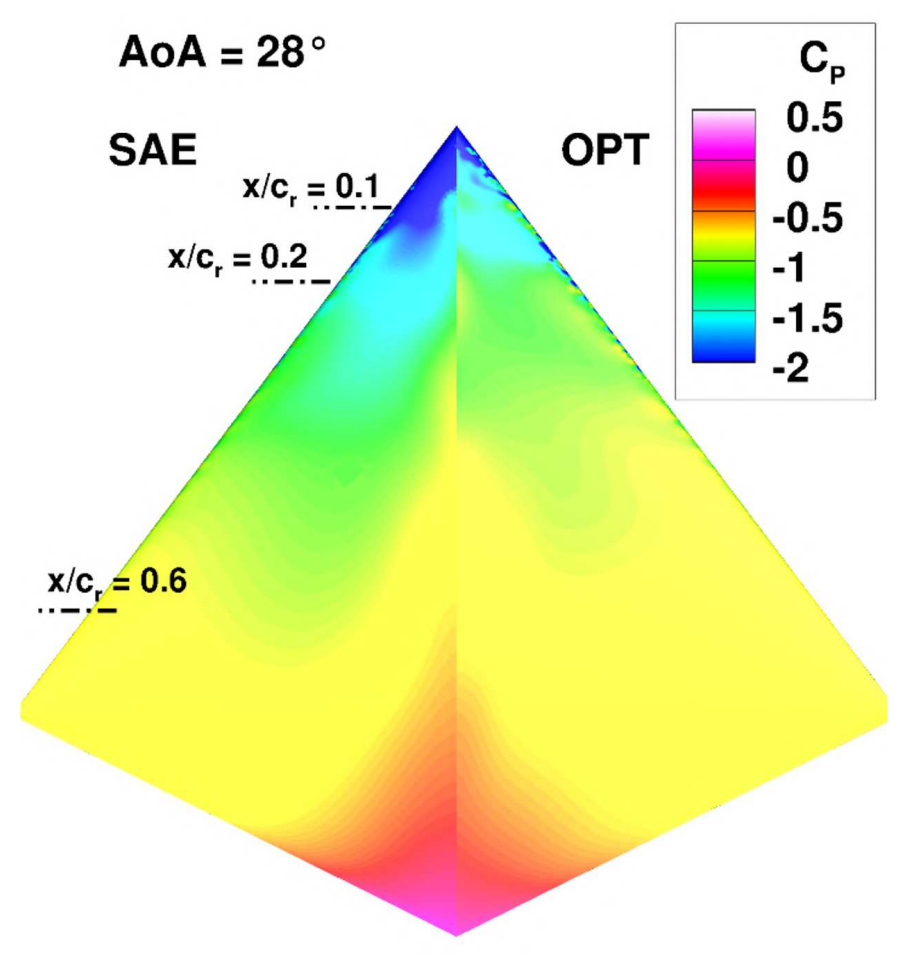

Figure 8: $C_{P}$ surface contour plot of the AVT-183 diamond wing at the flow conditions of $\mathrm{M}=0.15, \mathrm{Re}$ $=2.7 \cdot 10^{6}$ and $A o A=28^{\circ}$. The numerical results of the baseline (SAE) and the optimized model (OPT) (Tab. 3) are compared.

difference is reasonable due to the mentioned geometrical differences, in particular, the deployed slat on the Model53 provides a more stable vortex which delays the breakdown instability phenomenon. The application of the optimized set of turbulence model coefficients on the Model53 provides a similar variation of the vortex flow stage and a comparable improvement of the prediction to the experimental data. In fact, not only the vortex stage is now correctly predicted as a stall condition is achieved but the $C_{P}$ values show only small discrepancies compared to the experimental data downstream.

The angle of attack of $32^{\circ}$ is characterized by the presence of a stall condition already with the baseline SAE model and a flat $C_{P}$ distribution is evident. However, the suction level is larger compared to the experimental data as spurious tangential and rotational velocities are not dissipated through the shedding of the vortex directly at the leading edge and provide an additional suction to the wing surface. The application of the optimized model do improve the suction level to a certain amount but the accuracy improvement with the experimental data is fairly lower compared to the $28^{\circ}$ angle of attack case.

As second step, the ANN method is applied to the case of $A o A=32$ as target. The set of coefficients predicted by the ANN model (Tab. 7) is then applied for a numerical simulation. The contour surface plot is visualized in comparison to the SAE model in Figure 9 and it shows how the correct stall condition is still predicted by the machine learning (ML). Moreover, the suction distribution is better than the SAE baseline model as the spurious velocities of the stalled flow which maintain a higher suction level above the wing are better dissipated and the level of suction is closer to the experimental data (Figure 9). 

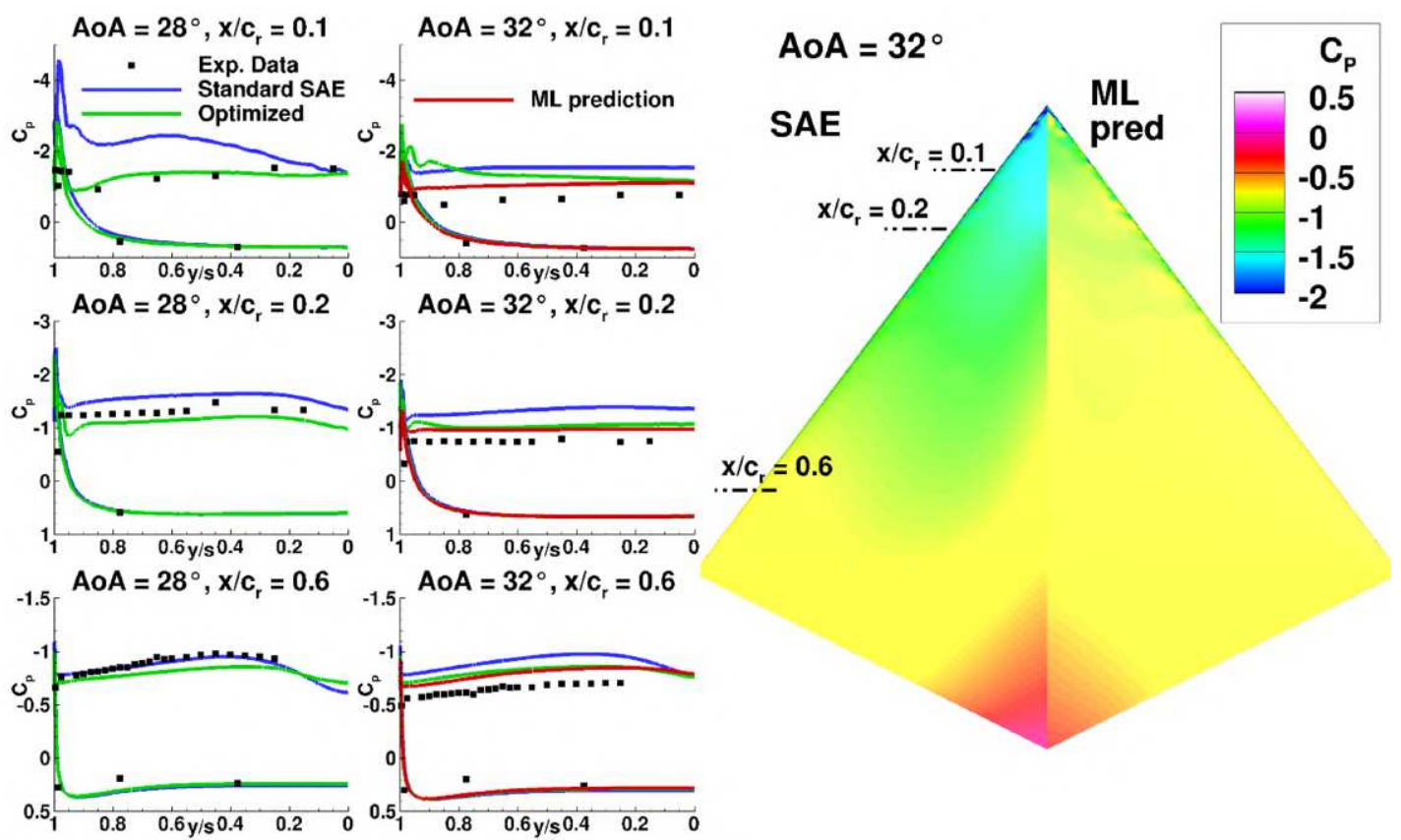

Figure 9: $C_{P}$ surface contour plot and sectional $C_{p}$ distributions of the AVT-183 diamond wing at the flow conditions of $\mathrm{M}=0.15, \mathrm{Re}=2.7 \cdot 10^{6}$ and $A o A=[28,32]^{\circ}$. The numerical results of the baseline (SAE), the model optimized on the Model53 (OPT) (Tab. 3) and the model predicted with the ANN methodology (ML) are compared to the experimental data (W/T Data).

The result is close to the accuracy improvement achieved with the optimized turbulence model on the Model53 for the region from $x / c_{r}=0.2$ and downwards. However, the first cross-section cut shows an even better prediction of the surface $C_{P}$ distribution than the Model53 optimum, because it is flatter and at lower suction level.

Table 7: Set of coefficients predicted with the ANN model for the AVT-183 diamond wing.

\begin{tabular}{c|ccc}
\hline Coefficient & $c_{b v 1}$ & $c_{b v h 1}$ & $c_{b v h 2}$ \\
\hline Value & 0.2479 & -0.02574 & 0.04235 \\
\hline
\end{tabular}

\subsubsection{SAGITTA Blended Wing Body Configuration}

As additional test case for the application of the ML methodology employing the ANN model, the SAGITTA blended wing body geometry is selected which consists of a $55^{\circ}$ swept diamond wing configuration $[19,20]$. The wind tunnel model is a full model and it has been mounted through a sting support which is also included in the numerical simulations. The measurements are performed at $M=0.1$ and $R e=1.77 \cdot 10^{6}$ based on the mean aerodynamic chord for the high angles of attack region. The test case employs some differences with regard to the other test cases reported in this work. The major differences are the larger sweep angle, the body thickness and also the leading-edge curvature which is rounded. With the application of the ML-methodology a slightly different set of coefficients is predicted (Tab. 9). 
The target angle of attack has been selected to be $36^{\circ}$.

Table 8: Blended Wing Body: wing geometry parameters.

\begin{tabular}{ccccccccc}
\hline$c_{r}[m]$ & $c_{t}[m]$ & $\lambda$ & $\varphi_{L E}$ & $\varphi_{T E}$ & $S_{r e f}\left[m^{2}\right]$ & $\Lambda$ & $l_{\mu}[m]$ & $r / c_{r}$ \\
\hline 1.2 & 0.03 & - & $55^{\circ}$ & $-25^{\circ}$ & 0.759 & 2.001 & var height &
\end{tabular}

Table 9: Set of coefficients predicted with the ANN model for the blended wing body.

\begin{tabular}{c|ccc}
\hline Coefficient & $c_{b v 1}$ & $c_{b v h 1}$ & $c_{b v h 2}$ \\
\hline Value & 0.23378 & -0.001 & -0.0403 \\
\hline
\end{tabular}

At $\mathrm{AoA}=36^{\circ}$ the vortex flow predicted by the SAE baseline model consists of a fully developed vortex with breakdown in the region between $x / c_{r}=0.3$ and 0.4 (Fig. 10). The SAE turbulence model shows a larger suction peak compared to the experimental data in the first two cross-flow sections whereas downstream of breakdown the accuracy is quite good. The experimental data also manifests the presence of the same vortex flow stage and not a full stall condition as it was the case of the calibration target of the Model53. The application of the ML-predicted set of coefficients improves the accuracy of the vortex flow related to the experimental data without changing the vortex flow stage. The suction footprint is sensitively reduced in the front part of the wing and the vortex flow acquires a physical oscillating nature in contrast to the baseline model. This phenomenon indicates that the additional vortex destruction of the turbulence model reduces the dissipation effect of the large eddy viscosity quantities produced with the baseline SAE model. Hence, the lower dissipation model produces unsteady flow phenomena and an oscillation of convergence which is physical in the case of highly unstable vortex flows at high angles of attack. In general, the accuracy improvement of this test case is appreciable and comparable with the other test cases. This provides an additional confirmation of the possibility to use the data available to extend the predictive capability of the methodology.

\section{CONCLUSION AND OUTLOOK}

The present results show how the turbulence model modification introducing additional source terms exclusively active in the vortex flow regions manifests the properties set as objective of its formulation and development. Hence, it does not introduce numerical instabilities or significant additional computational costs. Moreover, it is able to influence the vortex flow field only without worsening regions of attached flow where the baseline model is already well calibrated. The different additional turbulence terms are able to influence with flexibility and variable intensity different types and regions of the vortex flow development.

The methodology is applied to a generic $53^{\circ}$ swept delta wing, called Model53, and it has shown a consistent accuracy improvement throughout the angle-of-attack polar. The range of predictive capability is, therefore, investigated by means of different steps. First of all, the optimized model is applied to a close validation case which is the Model56 that consists of a sweep angle variation of $+3^{\circ}$. The validation case confirms a comparable grade of accuracy improvement of the calibration case. Second, the model is applied to a cluster of available test cases which share partial similarities to the calibration case with respect to their geometries and the class of vortex flow. The application of the previously optimized 

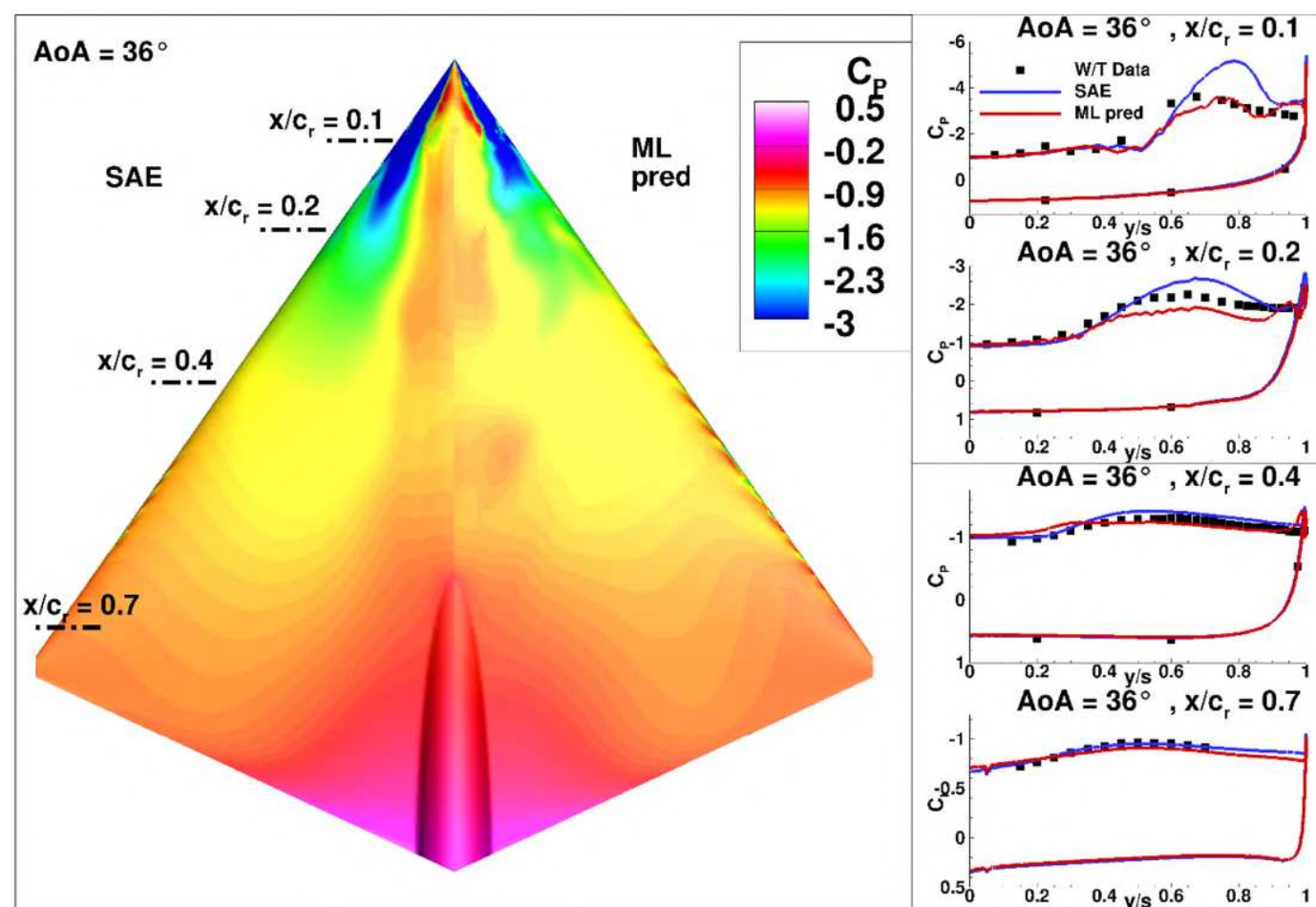

$\mathrm{AOA}=36^{\circ}, \mathrm{x} / \mathrm{c}_{\mathrm{r}}=0.4$

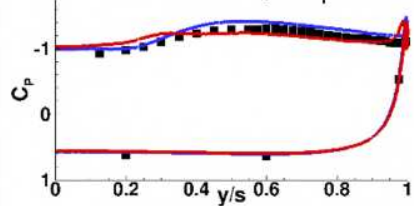

$\mathrm{AOA}=36^{\circ}, \mathrm{x} / \mathrm{c}_{\mathrm{r}}=0.7$

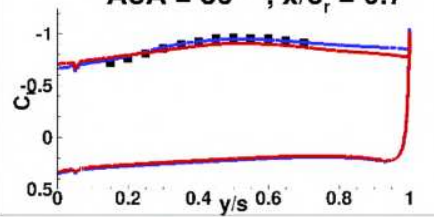

Figure 10: $C_{P}$ surface contour plot and sectional $C_{p}$ distribution of the Blended Wing Body at the flow conditions of $M=0.1, R e=1.77 \cdot 10^{6}$ and $A o A=36^{\circ}$. The numerical results of the baseline (SAE) and the model predicted with the ANN methodology (ML) are compared to the experimental data (W/T Data).

model show consistent improvements with respect to these additional cases. This confirms the potential of the methodology to be applied for a significant extension of geometries and flow conditions around the calibration case.

An important drawback is highlighted which consists of the limitation of the calibrated model which reduces its accuracy improvement if a significant difference in terms of geometry and flow condition is investigated. Hence, if a new test case needs to be investigated and no experimental data is available, the methodology might lack of efficient utility. For this reason, an additional methodology is introduced which employs an artificial neural network which is trained on all the available data from previous optimization procedures in order to predict a proper model calibration for a new test case in relation to its geometry and flow condition parameters. The methodology is applied on the additional validation test cases reported in this work, showing promising improvements with respect to the vortex flow development and accuracy related to the experimental data.

As an outlook to the research, additional test cases are ongoing to be investigated to further address the potential of the methodology for different flow cases. Moreover, the machine learning extension can be further improved by means of increase the complexity of the neural network and the amount of data in parallel. 


\section{ACKNOWLEDGMENTS}

The funding of parts of these investigations within the LUFO VI-1 project DIGIfly-I (Digital Flight of Air Vehicles - Adaptive turbulence model with neural network conditioning applied to wing flows featuring leading edge vortex systems, FKZ: 20X1909I) by the German Federal Ministry for Economic Affairs and Energy (BMWi) is gratefully acknowledged. Furthermore, the authors thank Airbus Defence and Space for the fruitful cooperation and the German Aerospace Center (DLR) for providing the DLR TAU-Code to conduct the numerical simulations. Moreover, the authors gratefully acknowledge the Gauss Centre for Supercomputing e.V. (www.gauss-centre.eu) for funding this project by providing computing time on the GCS Supercomputer SuperMUC-NG at Leibniz Supercomputing Center (LRZ, www.lrz.de).

\section{REFERENCES}

[1] Wu, J. and Xiao, H. and Sun, R. and Wang, Q. RANS Equations with Explicit Data-Driven Reynolds Stress Closure Can Be Ill-Conditioned. Journal of Fluid Mechanics, Vol. 869, 2019, pp. 553-586. 10.1017/jfm.2019.205.

[2] Freeman, J. Computational Fluid Dynamics Investigation of Vortex Breakdown for a Delta Wing at High Angle of Attack. Thesis, Department of the Air Force University, Air Force Institute of Technology, USAF, 2003.

[3] Spalart, P. R. and Allmaras, S. A One-Equation Turbulence Model for Aerodynamic Flows. AIAA, Vol. 439, 1992. 10.2514/6.1992-439.

[4] Moioli, M. and Breitsamter, C. and Sørensen, K.A. Parametric data-based turbulence modelling for vortex dominated flows. International Journal of Computational Fluid Dynamics, Vol. 33, No. 4, 2019, pp. 149-170. https://doi.org/10.1080/10618562.2019.1617857.

[5] Shur, M. L. and Strelets, M. K. and Travin, A. K. and Spalart, P. R. Turbulence Modeling in Rotating and Curved Channels: Assessing the Spalart-Shur Correction. AIAA Journal, Vol. 38, No. 5, 2000, pp. 784-792. 10.2514/2.1058.

[6] Zhang, X. and Agarwa, R. K. Development of Various Rotation and Curvature Corrections for Eddy-Viscosity Turbulence Models. AIAA Aerospace Sciences Meeting, 2018. 10.2514/6.20180591 .

[7] Duraisamy, K. and Iaccarino, G. and Xiao, H. Turbulence Modeling in the Age of Data. Annual Review of Fluid Mechanics, Vol. 51, No. 1, 2019, pp. 357-377. 10.1146/annurev-fluid-010518040547.

[8] Frink, N. T. Numerical Analysis of Incipient Separation on 53-Deg Swept Diamond Wing. 53rd AIAA Aerospace Sciences Meeting, 2015. 10.2514/6.2015-0288.

[9] Hummel, D. Experimental investigation of the flow on the suction side of a thin delta wing. NASA Technical Memorandum 75897, 1981.

[10] Breitsamter, C. Unsteady flow phenomena associated with leading-edge vortices. Progress in Aerospace Sciences, Vol. 44, No. 1, 2008, pp. 48 - 65. 10.1016/j.paerosci.2007.10.002.

[11] Hummel, D. On the Vortex Formation over a Slender Wing at Larg Angles of Incidence. High Angle of Attack Aerodynamics, CP-247, AGARD, 1979, pp. 15-1-15-17. 
[12] Lambourne, N. C. and Bryer, D. W. The bursting of leading-edge vortices-Some observations and discussion of the phenomenon.. ARCR \& M., Vol. 3282, 1962, pp. 1-36.

[13] Sørensen, K. A. VitAMin-ABC, Overview of Airbus D\&S activities in VitAM. (2018).

[14] Edwards, J. R. and Chandra, S. Comparison of eddy viscosity-transport turbulence models for three-dimensional, shock-separated flowfields. AIAA Journal, Vol. 34, No. 4, 1996, pp. 756-163. 10.2514/3.13137.

[15] Abadi, M. and Barham, P. and Chen, J. and Chen, Z. and Davis, A. and Dean, J. and Devin, M. and Ghemawat, S. and Irving, G. and Isard, M. and others Tensorflow: A system for large-scale machine learning. 2016, pp. 265-283. https://arxiv.org/abs/1605.08695.

[16] Moioli, M. and Reinbold, C. and Sørensen, K. A. and Breitsamter, C. Investigation of Additively Manufactured Wind Tunnel Models with Integrated Pressure Taps for Vortex Flow Analysis. Aerospace, Vol. 6, 2019, pp. 113. 10.3390/aerospace6100113.

[17] Luckring, J. M. and Boelens, O. J. and Breitsamter, C. and Hövelmann, A. and Knoth, F. and Malloy, D. J. and Deck, S. Objectives, approach, and scope for the AVT-183 diamondwing investigations. Aerospace Science and Technology, Vol. 57, No., 2016, pp. 2 - 17. 10.1016/j.ast.2016.05.025.

[18] Frink, N. T. Numerical Analysis of Incipient Separation on 53-Deg Swept Diamond Wing. 53rd AIAA Aerospace Sciences Meeting, 2015. 10.2514/6.2015-0288.

[19] Hövelmann, A. Analysis and Control of Partly-Developed Leading-Edge Vortices. Technische Universität München, Dissertation, Vol. 2016.

[20] Pfnür, S. and Oppelt, S. and Breitsamter, C. Yaw-control efficiency analysis for a diamond wing configuration with outboard split flaps. CEAS Aeronautical Journal, Vol. 10, 2019, pp. 645-663. 10.1007/s13272-018-0340-1. 